\title{
A complete sample of GHz-peaked-spectrum radio sources and its radio properties
}

\author{
C. Stanghellini ${ }^{1,5}$, C.P. O'Dea ${ }^{2}$, D. Dallacasa ${ }^{4}$, S.A. Baum ${ }^{2}$, R. Fanti ${ }^{3,4}$, and C. Fanti ${ }^{3,4}$ \\ 1 Istituto di Radioastronomia del CNR, C.P. 141, I-96017 Noto SR, Italy \\ 2 Space Telescope Science Institute, 3700 San Martin Drive, Baltimore, MD 21218, U.S.A. \\ 3 Dipartimento di Fisica, Università degli Studi, Via Irnerio 46, I-40126 Bologna, Italy \\ 4 Istituto di Radioastronomia del CNR, Via Gobetti 101, I-40100 Bologna, Italy \\ 5 Visitor at the Space Telescope Science Institute, Baltimore
}

Received January 5; accepted March 16, 1998

\begin{abstract}
We define a complete sample of thirty-three GHz-Peaked-Spectrum (GPS) radio sources based on their spectral properties. We present measurements of the radio spectra and polarization of the complete sample and a list of additional GPS sources which fail one or more criteria to be included in the complete sample.

The majority of the data have been obtained from quasi-simultaneous multi-frequency observations at the Very Large Array (VLA) during 3 observing sessions. Low frequency data from the Westerbork Synthesis Radio Telescope (WSRT) and from the literature have been combined with the VLA data in order to better define the spectral shape.

The objects presented here show a rather wide range of spectral indices at high and low frequencies, including a few cases where the spectral index below the turnover is close to the theoretical value of 2.5 typical of self-absorbed incoherent synchrotron emission. Faint and diffuse extended emission is found in about $10 \%$ of the sources.

In the majority of the GPS sources, the fractional polarization is found to be very low, consistent with the residual instrumental polarization of $0.3 \%^{1}$.
\end{abstract}

Key words: galaxies: active — quasars: general — radio continuum: galaxies

\section{Introduction}

The GHz-peaked-spectrum (GPS) radio sources are characterized by a simple convex spectrum which peaks in

Send offprint requests to: C. Stanghellini

1 Tables 4 and 5 are only available in electronic form at the CDS via anonymous ftp to cdsarc.u-strasbg.fr (130.79.128.5) or via http://cdsweb.u-strasbg.fr/Abstract.html a range of about a decade around $1 \mathrm{GHz}$. General discussions on the general properties of these objects are given by O'Dea et al. (1991) O'Dea \& Baum (1997), and O'Dea (1998) where an exhaustive bibliography can also be found.

Common characteristics of the bright sample of GPS radio sources are: small size $(\lesssim 1 \mathrm{kpc})$, high radio luminosity, low fractional polarization, and apparently low variability. They are a mixed class of quasars and galaxies. Galaxies tend to be $L_{*}$ or brighter and at redshifts $0.1 \lesssim z \lesssim 1$ (O'Dea et al. 1996) while quasars are often found at very large redshift $1 \lesssim z \lesssim 4$, (O'Dea 1990).

Currently, there are two main competing hypotheses to explain the origin of GPS radio sources and their possible evolution.

In the "young source" scenario, first suggested by Phillips \& Mutel (1982), GPS radio sources with compact double (CD) morphology or compact symmetric morphology (Compact Symmetric Objects: CSO) are classical double radio sources at the very first stage of their lives.

In the "frustration" scenario, GPS radio sources will never become as large as the classical doubles since they are confined to the sub-kpc scale by a dense and turbulent ambient medium (e.g., O'Dea et al. 1991; Carvalho 1994, 1998). However, it is unclear whether the gas density and its distribution in the nuclear region is sufficient to confine the radio source for times of the order of $10^{7}$ years (see e.g., O'Dea 1998).

Recent results support the young source hypothesis. Fanti et al. (1995) presented a model for the evolution of the galactic-scale Compact Steep Spectrum (CSS) sources into large scale classical doubles. They argued that the objects showing symmetric morphology are probably not confined by a dense and clumpy medium (see also De Young 1993). They also suggest that the typical CSS source age is of the order of $10^{6}$ years and that the 
radio source luminosity decreases by an order of magnitude as the size of the radio source grows from a few kpc to hundreds of kpc (see also Begelman 1996). The same conclusions are reached by Readhead et al. $(1996 \mathrm{a}, \mathrm{b})$ in their study of a few CSO's. O'Dea \& Baum (1997), combining the complete sample of GPS presented here with the CSS sources by Fanti et al. (1990) reach similar conclusions. They further note that the luminosity evolution required makes it likely that GPS and CSS radio sources will evolve into objects less powerful than the most powerful classical doubles.

The detection of arc-second scale faint extended emission around $\sim 10 \%$ of GPS sources (Baum et al. 1990; Stanghellini et al. 1990) motivated Baum et al. (1990) to suggest that nuclear activity is recurrent in these sources. In this hypothesis, we see the relic of a previous epoch of activity as faint diffuse emission surrounding the current young nuclear source.

In order to achieve a deeper understanding of the GPS radio sources we pursued the following project.

(1) We created a complete sample of GPS radio sources by means of a preliminary bibliographic research, checked with subsequent new multi-frequency data from the Very Large Array (VLA) and the Westerbork Synthesis Radio Telescope (WSRT).

(2) We determined the properties of the radio spectrum and the polarization.

(3) We obtained optical imaging to determine the host galaxy properties.

(4) We obtained VLBI observations to study the milliarcsecond radio morphology.

In this paper we present the selected sample and the results from the VLA and WSRT radio observations. The optical properties of GPS radio sources are discussed by O'Dea et al. (1990), Stanghellini et al. (1993), and O'Dea et al. (1996). The milliarcsecond morphology is presented in Stanghellini et al. (1997). Constraints on radio source evolution based on our observations of the complete GPS sample are discussed by O'Dea \& Baum (1997).

$H_{0}=100 \mathrm{~km} \mathrm{~s}^{-1} \mathrm{Mpc}^{-1}$, and $q_{0}=0.5$ have been used in this paper.

\section{The complete sample}

Research on GPS radio sources has increased in the past few years and a heterogeneous list of objects has been accumulated. The list presented by O'Dea et al. (1991) consists of $\sim 100$ radio sources, while Dallacasa \& Stanghellini (1990) collected a larger list of GPS and CSS radio sources and candidates. To enable statistical analysis of the properties of these objects we selected a complete sample of bright GPS radio sources.

The selection criteria were:

- declination $\delta>-25^{\circ}$

- galactic latitude $|l|>10^{\circ}$
- flux density at $5 \mathrm{GHz} S_{5 \mathrm{GHz}}>1 \mathrm{Jy}$

- turnover frequency between 0.4 and $6 \mathrm{GHz}$

- spectral index $\alpha_{\text {thin }}>0.5\left(S_{\nu} \propto \nu^{-\alpha}\right)$ in the high frequency, likely optically thin, part of the spectrum.

We started by selecting GPS candidates from the $1 \mathrm{Jy}$ catalog of Kühr et al. (1981). We cleaned this first "dirty" sample (Stanghellini et al. 1990) using our multi-frequency observations from the VLA and the WSRT presented here, supplemented with data from the literature.

The final complete sample consists of the 33 objects listed in Table 1. This is the first complete sample of bright GPS objects. The new radio data permit the proper classification and the improvement in the estimate of important parameters such as spectral indices, turnover frequencies, and polarization properties.

We note the following regarding the sample and possible selection effects.

Since variability may influence the spectral shape, the fact that GPS sources are thought to not be significantly variable may be just due to selection effects. Even if the spectral shape is constant, if the flux density varies significantly, the source would probably not be recognized as GPS using data from the literature spanning several years. Therefore it is important to have simultaneous multifrequency observations to build a sample unbiased with respect to variability (see also Sect. 4.4).

The turnover frequency and the spectral indices at low and high frequencies are difficult to derive when few data are available and/or the spectrum bends continuously. For this reason we included objects with a turnover frequency in a range a little larger than that of the canonical decade around $1 \mathrm{GHz}$.

The selection is based on the observed turnover frequency, hence we select objects with different intrinsic turnover frequencies at low and high redshifts. A selection based on the intrinsic turnover frequency is not currently possible due to the lack of complete redshift information on large samples of radio sources.

We note that the sample is unbiased regarding the optical identification.

\section{The observations}

The flux densities obtained in the different observing sessions are presented in Tables 4 and 5. Selected data from the literature have been added (with the relevant references) when considered compatible with our observations and useful to define the spectral shape.

During the VLA observing sessions an additional 20 GPS candidate sources were observed (Table 2). These sources fail one or more criteria of the complete sample. Nevertheless, the majority of them show a clear GPS-like spectrum. 
Table 1. Complete sample: Cols. 1 to 10, source name, redshift, optical identification, magnitude and filter/color, flux density at $5 \mathrm{GHz}$, observed and rest frame peak frequency, references for the optical information 1) O'Dea et al. 1991; 2) de Vries et al. 1995; 3) Stanghellini et al. 1993; 4) Snellen et al. 1996; 5) Stanghellini et al. 1997; 6) de Vries (private communication); 7) White et al. 1993; 8) Hewitt \& Burbidge 1987; 9) Hunter et al. 1993; 10) Heckman et al. 1994; 11) Stickel \& Kühr 1993a; 12) O'Dea et al. 1996; 13) Stickel \& Kühr 1993b; 14) Junkkarinen et al. 1991. Last column is a note indicating the radio source with extended emission

\begin{tabular}{|c|c|c|c|c|c|c|c|c|c|c|c|}
\hline name & $z$ & id. & $m$ & $S_{5 \mathrm{GHz}}$ & $\nu_{\text {to }}$ & $\nu_{\text {rest }}$ & $\alpha_{\text {low } \nu}$ & $\alpha_{\text {high } \nu}$ & $S_{\max }$ & ref. & note \\
\hline 0019-000 & 0.305 & $\mathrm{G}$ & $18.4 \mathrm{r}$ & 1.10 & 0.8 & 1.0 & -1.0 & 1.2 & 3.47 & 3,4 & \\
\hline $0108+388$ & 0.669 & $\mathrm{G}$ & $22.0 \mathrm{r}$ & 1.29 & 3.9 & 6.5 & -2.1 & 0.9 & 1.33 & 1,3 & $\mathrm{e}$ \\
\hline $0237-233$ & 2.223 & Q & $16.6 \mathrm{~V}$ & 3.34 & 1.0 & 3.2 & -1.9 & 0.7 & 7.05 & 1 & \\
\hline $0248+430$ & 1.316 & $\mathrm{Q}$ & $15.5 \mathrm{~V}$ & 1.24 & 5.2 & 12.1 & -0.4 & 0.6 & 1.27 & 1 & e? \\
\hline $0316+161$ & $\ldots$ & G & $23.6 \mathrm{R}$ & 2.91 & 0.8 & 1.2 & -0.7 & 1.1 & 9.55 & 3 & \\
\hline $0428+205$ & 0.219 & $\mathrm{G}$ & $19.3 \mathrm{R}$ & 2.30 & 1.0 & 1.3 & -0.6 & 0.6 & 4.02 & 1,3 & \\
\hline $0457+024$ & 2.384 & $\mathrm{Q}$ & $19.4 \mathrm{~V}$ & 1.57 & 1.9 & 6.3 & -3.5 & 0.6 & 1.89 & 1 & \\
\hline $0500+019$ & 0.583 & $\mathrm{G}$ & $21.0 \mathrm{i}$ & 1.89 & 2.0 & 3.2 & -1.6 & 0.9 & 2.51 & 2 & \\
\hline $0710+439$ & 0.518 & $\mathrm{G}$ & $18.5 \mathrm{i}$ & 1.67 & 1.9 & 2.8 & -1.3 & 0.6 & 2.09 & 1,3 & \\
\hline $0738+313$ & 0.631 & $\mathrm{Q}$ & $16.1 \mathrm{~V}$ & 3.66 & 5.1 & 8.3 & -0.7 & 0.8 & 3.82 & 1 & $\mathrm{e}$ \\
\hline $0742+103$ & $\ldots$ & $\mathrm{S} ?$ & $23 \mathrm{r}$ & 3.46 & 2.8 & 5.7 & -0.7 & 0.7 & 4.12 & 1,5 & \\
\hline 0743-006 & 0.994 & $\mathrm{Q}$ & $17.5 \mathrm{~V}$ & 2.05 & 6.0 & 12.0 & -0.6 & 0.8 & 2.12 & 1,5 & \\
\hline 0941-080 & 0.228 & $\mathrm{G}$ & $17.9 \mathrm{r}$ & 1.10 & 0.5 & 0.6 & -0.3 & 1.0 & 3.40 & 3,6 & e? \\
\hline $1031+567$ & 0.459 & $\mathrm{G}$ & $20.2 \mathrm{r}$ & 1.27 & 1.3 & 1.8 & -0.7 & 0.8 & 1.87 & 1,3 & \\
\hline $1117+146$ & 0.362 & G & $20.1 \mathrm{R}$ & 1.00 & 0.5 & 0.7 & -0.4 & 0.8 & 3.89 & 1,3 & \\
\hline $1127-145$ & 1.187 & $\mathrm{Q}$ & $16.9 \mathrm{~V}$ & 3.82 & 1.0 & 2.2 & -0.8 & 0.6 & 5.80 & 8 & \\
\hline $1143-245$ & 1.95 & $\mathrm{Q}$ & $18.5 \mathrm{~V}$ & 1.40 & 2.0 & 5.9 & -2.5 & 0.7 & 1.69 & 1 & \\
\hline $1245-197$ & 1.273 & $\mathrm{Q}$ & $20.5 \mathrm{~V}$ & 2.31 & 0.5 & 1.2 & -0.7 & 0.9 & 8.69 & 1 & \\
\hline $1323+321$ & 0.369 & $\mathrm{G}$ & $19.2 \mathrm{r}$ & 2.35 & 0.5 & 0.7 & -0.7 & 0.6 & 7.03 & 1,3 & \\
\hline $1345+125$ & 0.122 & G & $15.5 \mathrm{r}$ & 3.03 & 0.6 & 0.6 & -0.9 & 0.7 & 8.86 & 1,3 & \\
\hline $1358+624$ & 0.431 & $\mathrm{G}$ & $19.8 \mathrm{r}$ & 1.80 & 0.5 & 0.8 & -1.5 & 0.7 & 6.56 & 1,3 & \\
\hline $1404+286$ & 0.077 & Sy & $14.6 \mathrm{r}$ & 2.66 & 4.9 & 5.3 & -1.5 & 1.6 & 2.76 & 1,3 & \\
\hline $1442+101$ & 3.544 & $\mathrm{Q}$ & $17.8 \mathrm{~V}$ & 1.19 & 0.9 & 4.1 & -1.3 & 0.9 & 2.61 & 1 & \\
\hline $1518+047$ & 1.296 & $\mathrm{Q}$ & $22.6 \mathrm{r}$ & 1.05 & 0.9 & 2.1 & -0.6 & 1.3 & 4.58 & 1,10 & \\
\hline $1600+335$ & $\ldots$ & G & $23.2 \mathrm{r}$ & 2.67 & 2.6 & 3.9 & -0.2 & 0.9 & 3.06 & 1,3 & \\
\hline $1607+268$ & 0.473 & $\mathrm{G}$ & $20.4 \mathrm{r}$ & 1.71 & 1.0 & 1.5 & -1.5 & 1.2 & 5.44 & 1,3 & \\
\hline $2008-068$ & $\ldots$ & $\mathrm{G}$ & $21.3 \mathrm{R}$ & 1.34 & 1.3 & 2.0 & -0.9 & 0.8 & 2.64 & 1,3 & \\
\hline $2126-158$ & 3.270 & $\mathrm{Q}$ & $17.3 \mathrm{~V}$ & 1.17 & 3.9 & 16.7 & -1.3 & 0.5 & 1.23 & 1 & \\
\hline $2128+048$ & 0.99 & $\mathrm{G}$ & $23.3 \mathrm{r}$ & 2.02 & 0.8 & 1.6 & -1.0 & 0.8 & 4.93 & 1,11 & \\
\hline $2134+004$ & 1.936 & $\mathrm{Q}$ & $16.8 \mathrm{~V}$ & 8.50 & 5.2 & 15.4 & -1.2 & 0.7 & 8.59 & 8 & $\mathrm{e}$ \\
\hline $2210+016$ & $\ldots$ & G & $22.0 \mathrm{i}$ & 1.05 & 0.4 & 0.6 & -0.6 & 1.0 & 4.51 & 2 & \\
\hline $2342+821$ & 0.735 & Q & $20.1 \mathrm{r}$ & 1.28 & 0.5 & 0.8 & -0.7 & 0.9 & 6.29 & 1,3 & \\
\hline $2352+495$ & 0.237 & $\mathrm{G}$ & $18.4 \mathrm{R}$ & 1.47 & 0.7 & 0.8 & -0.2 & 0.5 & 2.93 & 1 & \\
\hline
\end{tabular}

\subsection{VLA observations}

The VLA data were obtained in a number of observing sessions in the A configuration spanning several years. Eighteen candidate GPS radio sources were observed on 30 December 1984. The results from this first set of observations were already published by O'Dea et al. (1990), Stanghellini et al. (1990), and O'Dea et al. (1991). We consider here only the observed sources belonging to the complete sample.

A second set of VLA observations was obtained on 9 February 1990, and a third session on 8 June 1991. The journal of these observations in shown in Table 3 . In the third session, where the majority of the sources belonging to the complete sample were observed, we split the 2 frequencies in each band to obtain the maximum possible separation with adequate sensitivity. This was used in the previous observing sessions only in the $L$ band, here we used it also in the $C, X$ and $P$ bands. This technique proved to be very useful, because it permitted a better definition of the spectral shape and a more accurate selection of the sources peaking close to the limit of $0.4 \mathrm{GHz}$ that we adopted for our sample.

Furthermore, the observations separated in frequency permit us to solve the $n \pi$ ambiguity that occurs in the determination of the Faraday rotation measure.

The data reduction has been performed in a uniform way for all the VLA data sets. The errors in the flux densities are dominated by the calibration errors which are 
Table 2. Additional objects: Cols. 1 to 10, source name, redshift, optical identification, magnitude and filter/color, flux density at $5 \mathrm{GHz}$, observed and rest frame peak frequency, references for the optical information, references for the optical information as Table 1. Last column is a note indicating the radio source with extended emission

\begin{tabular}{cccccccccccc}
\hline name & $z$ & id. & $m$ & $S_{5 \mathrm{GHz}}$ & $\nu_{\text {to }}$ & $\nu_{\text {rest }}$ & $\alpha_{\text {low } \nu}$ & $\alpha_{\text {high } \nu}$ & $S_{\text {max }}$ & ref. & note \\
\hline $0026+346$ & 0.6 & $\mathrm{G}$ & $21.0 \mathrm{r}$ & 1.31 & 1.4 & 2.3 & -0.2 & 0.4 & 1.90 & 4 & \\
$0201+113$ & 3.61 & $\mathrm{Q}$ & $19.5 \mathrm{R}$ & 0.837 & $\ldots$ & $\ldots$ & -0.6 & 0.2 & $\ldots$ & 1,7 & \\
$0404+768$ & 0.599 & $\mathrm{G}$ & $21.1 \mathrm{r}$ & 2.91 & 0.3 & 0.5 & -0.3 & 0.5 & 8.15 & 1,3 & \\
$0440-003$ & 0.844 & $\mathrm{Q}$ & $19.22 \mathrm{~V}$ & 1.13 & 0.3 & 0.6 & -0.3 & 0.1 & 1.21 & 8 & \\
$0528+134$ & 2.07 & $\mathrm{Q}$ & $19.2 \mathrm{r}$ & 1.96 & 1.9 & 5.74 & -0.4 & 0.1 & 2.14 & 3,9 & $\mathrm{e}$ \\
$0552+398$ & 2.365 & $\mathrm{Q}$ & $18.0 \mathrm{~V}$ & 6.31 & 9.5 & 32.1 & -1.6 & 0.6 & 7.80 & 1 & \\
$0703+468$ & $\ldots$ & $\mathrm{S}$ & $23.1 \mathrm{r}$ & 0.640 & 0.7 & 1.4 & -1.7 & 1.0 & 2.37 & 1,3 & \\
$0711+356$ & 1.620 & $\mathrm{Q}$ & $17.0 \mathrm{~V}$ & 0.861 & 1.6 & 4.1 & -0.7 & 0.7 & 1.42 & 1 & \\
$0904+039$ & $\ldots$ & $\mathrm{E}$ & $\ldots$ & 0.216 & 0.6 & 1.3 & -0.4 & 1.3 & 1.04 & 1,2 & \\
$0914+114$ & $\ldots$ & $\mathrm{G}$ & $20.0 \mathrm{r}$ & 0.120 & 0.3 & 0.7 & -0.1 & 1.6 & 2.30 & 3 & \\
$1543+005$ & 0.550 & $\mathrm{G}$ & $20.0 \mathrm{R}$ & 1.23 & $\ldots$ & $\ldots$ & 0.1 & 0.5 & $\ldots$ & 1,10 & \\
$1732+094$ & $\ldots$ & $\mathrm{G}$ & $20.7 \mathrm{R}$ & 0.742 & 2.3 & 4.5 & -1.1 & 1.1 & 1.35 & 1 & \\
$2015+657$ & 2.845 & $\mathrm{Q}$ & $19.7 \mathrm{R}$ & 0.667 & $\ldots$ & $\ldots$ & -0.3 & 0.2 & $\ldots$ & 1,11 & \\
$2021+614$ & 0.2266 & $\mathrm{G}$ & $17.9 \mathrm{R}$ & 2.82 & 8.4 & 10.2 & -0.2 & 0.6 & 3.29 & 1 & \\
$2050+364$ & 0.354 & $\mathrm{G}$ & $21.2 \mathrm{r}$ & 3.28 & 2.1 & 2.9 & -0.4 & 0.9 & 5.82 & 1,12 & \\
$2137+209$ & 1.576 & $\mathrm{Q}$ & $19 \mathrm{R}$ & 0.590 & $\ldots$ & $\ldots$ & 0.3 & 0.9 & $\ldots$ & 1,10 & \\
$2149+056$ & 0.740 & $\mathrm{G}$ & $20.4 \mathrm{R}$ & 0.840 & 2.5 & 4.3 & -1.5 & 0.7 & 1.09 & 1,13 & \\
$2223+210$ & 1.949 & $\mathrm{Q}$ & $18.2 \mathrm{~V}$ & 1.07 & $\ldots$ & $\ldots$ & $\ldots$ & $\ldots$ & $\ldots$ & 1,14 & $\mathrm{e}$ \\
$2230+114$ & 1.037 & $\mathrm{Q}$ & $17.3 \mathrm{~V}$ & 4.12 & 0.5 & 1.0 & -0.7 & 0.4 & 8.35 & 1 & \\
$2337+264$ & $\ldots$ & $\mathrm{G}$ & $20.0 \mathrm{i}$ & 0.996 & 2.7 & 5.5 & -0.2 & 0.6 & 1.12 & 2 & \\
\hline
\end{tabular}

Table 3. Journal of the second and third VLA observing sessions. Only a few sources were observed at $22 \mathrm{GHz}$

\begin{tabular}{ccc}
\hline date & $\nu_{\mathrm{GHz}}$ & $\Delta \nu_{\mathrm{MHz}}$ \\
\hline 09 Feb. 1990 & 1.380 & 12.5 \\
09 Feb. 1990 & 1.630 & 12.5 \\
09 Feb. 1990 & 4.815 & 12.5 \\
09 Feb. 1990 & 4.865 & 12.5 \\
09 Feb. 1990 & 8.435 & 12.5 \\
09 Feb. 1990 & 8.485 & 12.5 \\
09 Feb. 1990 & 22.435 & 50 \\
09 Feb. 1990 & 22.485 & 50 \\
08 Jun. 1991 & 0.302 & 3 \\
08 Jun. 1991 & 0.333 & 3 \\
08 Jun. 1991 & 1.335 & 12.5 \\
08 Jun. 1991 & 1.665 & 12.5 \\
08 Jun. 1991 & 4.535 & 12.5 \\
08 Jun. 1991 & 4.985 & 12.5 \\
08 Jun. 1991 & 8.085 & 12.5 \\
08 Jun. 1991 & 8.465 & 12.5 \\
\hline
\end{tabular}

estimated to be around $3 \%$ at $L, C, X$ bands, around $5 \%$ at $P$ and $K$ bands, respectively.

At VLA resolution almost all the objects observed are dominated by a single point-like component. The side lobes have been deconvolved from the images using the AIPS implementation of the Clark CLEAN algorithm (Clark 1980; Cornwell \& Braun 1988). The data have been self-calibrated in phases (Schwab 1980; Cornwell \&
Fomalont 1988) with an initial point model, and when subsequent iterations of self-cal have been considered necessary, we used an appropriate number of non negative components obtained from the images in an interactive process until convergence to an acceptable solution was achieved. In most cases an amplitude self-calibration has been performed on the data to improve the final images and to allow a search for possible extended emission.

\subsection{The WSRT data}

Westerbork Synthesis Radio Telescope (WSRT) filler observations on most of the objects of the complete sample have been obtained during 1990 at $327 \mathrm{MHz}$ and during 1991 at $608 \mathrm{MHz}$ and $327 \mathrm{MHz}$. Each source has been observed in several snapshots a few tens of minutes long.

The data reduction for the data obtained in the 1990 has been done with the package DWARF. The data of the 1991 observations have been reduced with AIPS. These latter data in a format readable by AIPS did not have the redundant baselines, therefore the data were not selfcalibrated, producing a lower dynamic range compared with the capabilities of this instrument.

\subsection{Other data}

A few objects were observed during the spring and the summer of 1991 with the Northern Cross, a radio 


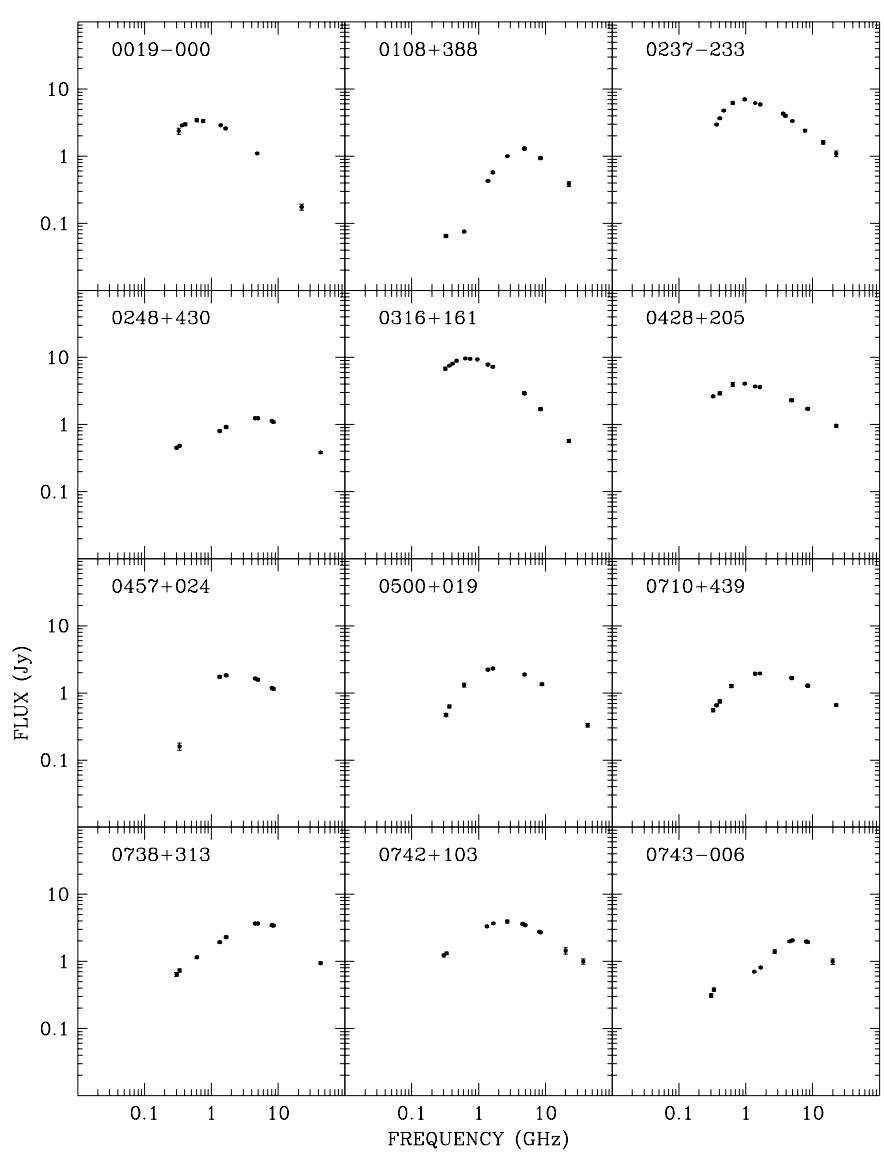

Fig. 1. Radio spectra of the objects of the complete sample

telescope near Bologna operating at $408 \mathrm{MHz}$ on the principle of the Mill's cross (Braccesi et al. 1969 and Ficarra et al. 1985). The radio telescope dates back to the sixties, however, recent mechanical and electronics upgrades allow completely automatic observations with increased sensitivity. The flux density values have been set to the scale of Baars (Baars et al. 1977) adopting a flux density of 37.7 Jy for the calibrator source 3C 380 (Riley 1988).

A few additional objects have been observed with the Russian radio telescope RATAN 600 (Esepkina et al. 1979), in transit mode, during various sessions in 1993 and 1994 , at $11.2,7.7,3.9,2.3$ and $0.96 \mathrm{GHz}$ (Mingaliev, private communication).

\section{Results and discussion}

In this paper we present the observational results and the primary qualitative conclusions. In a future paper we will present a quantitative discussion of the results.

\subsection{The radio spectra}

The flux densities are presented in Tables 4 and 5 and plotted in Figs. 1 to 5 .

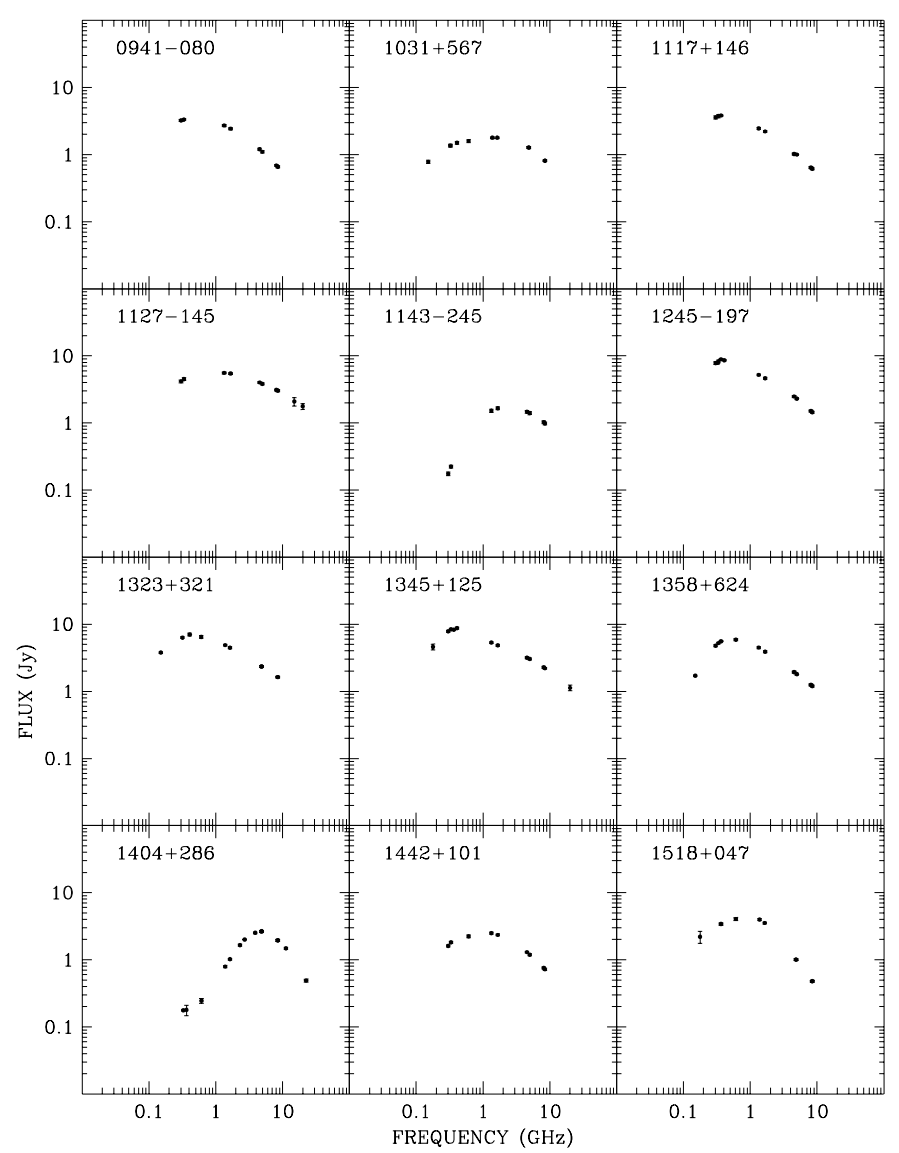

Fig. 2. Radio spectra of the objects of the complete sample

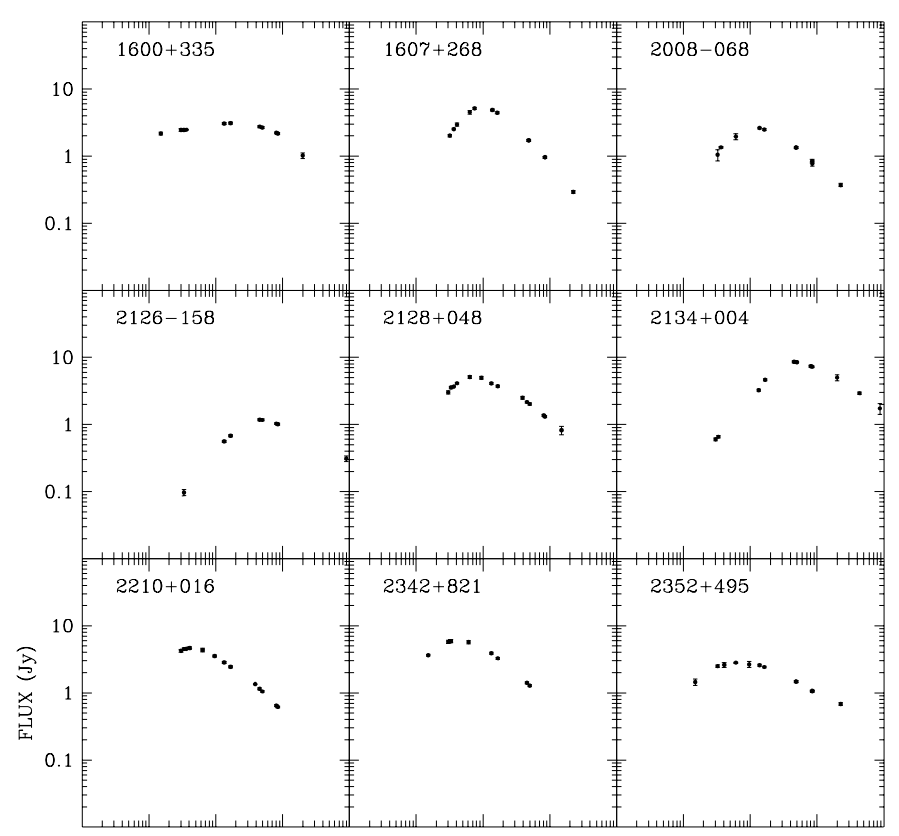

Fig. 3. Radio spectra of the objects of the complete sample 


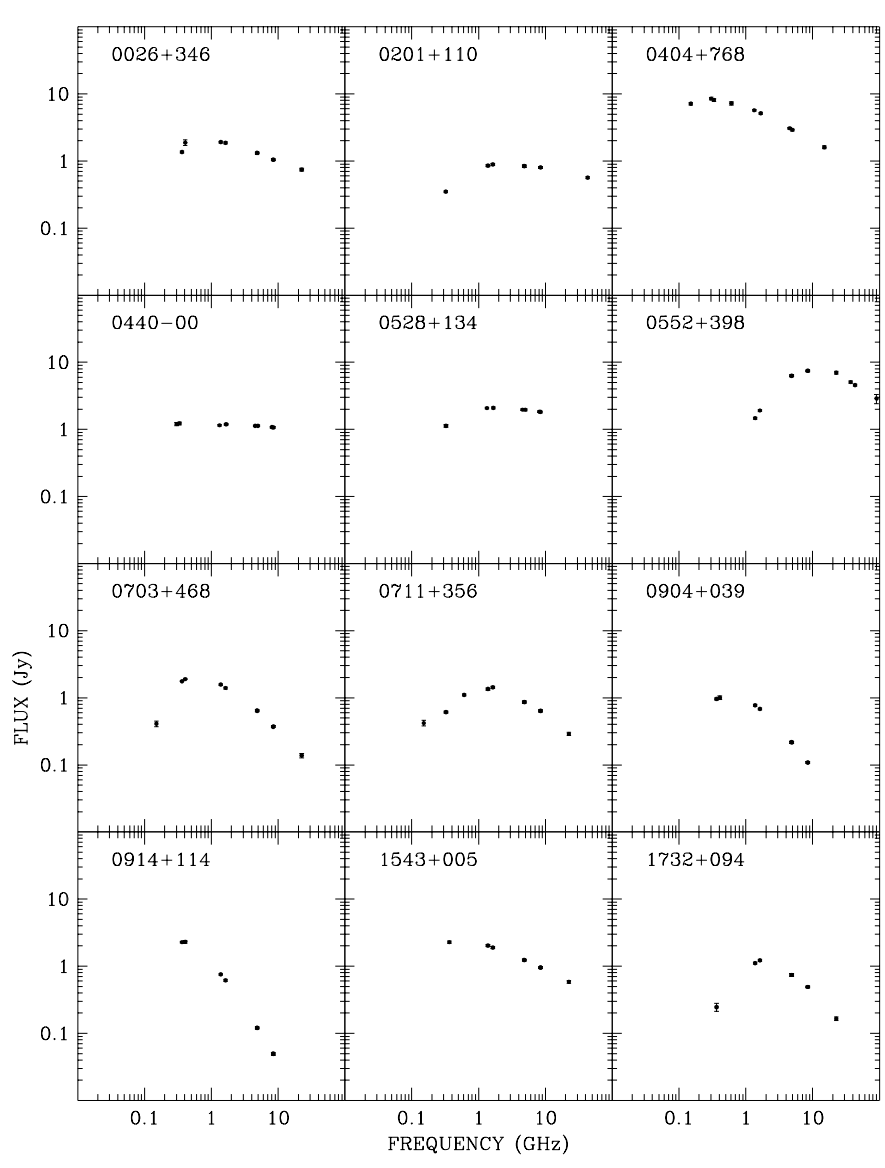

Fig. 4. radio spectra of the additional objects

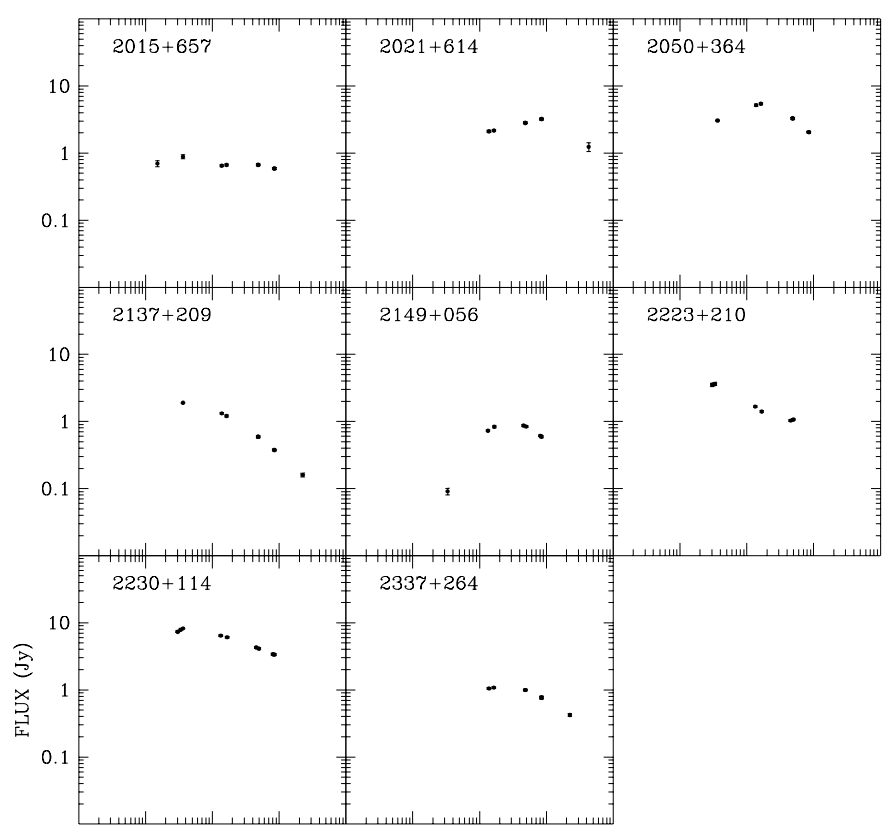

Fig. 5. radio spectra of the additional objects
In order to extract information from the radio spectra, we fitted them with a hyperbola (which is a curve tending asymptotically to a straight line at the extrema). We first fit the spectral indices in the thick and thin part of the spectrum independently, and then fixed these two values (which correspond to the angular coefficients of the asymptotes) and we performed a least square fit, solving for the other parameters of the hyperbola.

We calculated the frequency of the spectral peak (Tables 1 and 2) using the fitted curves. We show the distributions of the observed and rest frame turnover frequency for the 33 sources of the complete sample in Figs. $6 \mathrm{c}$ and $6 \mathrm{~d}$. In Figs. 6a and $6 \mathrm{~b}$ we show the high frequency (above the peak) and low frequency (below the peak) spectral index distribution, respectively.

\subsection{The spectral indices and the turnover frequency}

The GPS radio sources are a mixed group of galaxies and quasars, with some remarkable differences between the two classes. The histogram in Fig. 16a shows that the redshift distribution is very different for galaxies and quasars. The galaxies have a typical redshift of $\sim 0.5$, and none has a redshift higher than 1 . For the galaxies without redshift information, we note that only $0316+161$ has an optical magnitude slightly fainter than the galaxy $2128+048$ at redshift 0.99 (Table. 1). Since these galaxies follow the Hubble diagram (O'Dea et al. 1996; Snellen et al. 1996) it is unlikely they will be found at a redshift much higher than the others in the sample. The quasars are instead found at any redshift (we included the galaxy 1404+286 (OQ208), which has a Seyfert 1 nucleus, in the quasar class) with the majority at very high $z$ (see also O'Dea 1990).

The high frequency spectral index ranges from 0.5 (the limit set in the selection criteria) to 1.3 with the galaxies having perhaps slightly steeper values (but the 2 objects with the steepest spectral indices are quasars). The low frequency spectral index ranges from -0.2 to -2.1 without any clear difference between galaxies and quasars, but the higher values are biased since in several objects the low frequency part of the spectrum is under-sampled and the spectral index is calculated close to the turnover frequency where it is likely to be flatter. Similar results were found by De Vries et al. (1997) though their poorer frequency coverage resulted in a somewhat smaller range in spectral index.

The quasars tend to peak at higher frequencies than the galaxies in both the rest frame and observed frame and some quasars have a turnover frequency in their rest frame exceeding $10 \mathrm{GHz}$. This suggests that, on the assumption that the turnover is caused by synchrotron self-absorption, the GPS quasars are more compact than the galaxies. This effect has been also found by De Vries et al. (1997) in a bright heterogeneous sample and by Snellen (1997) in a 

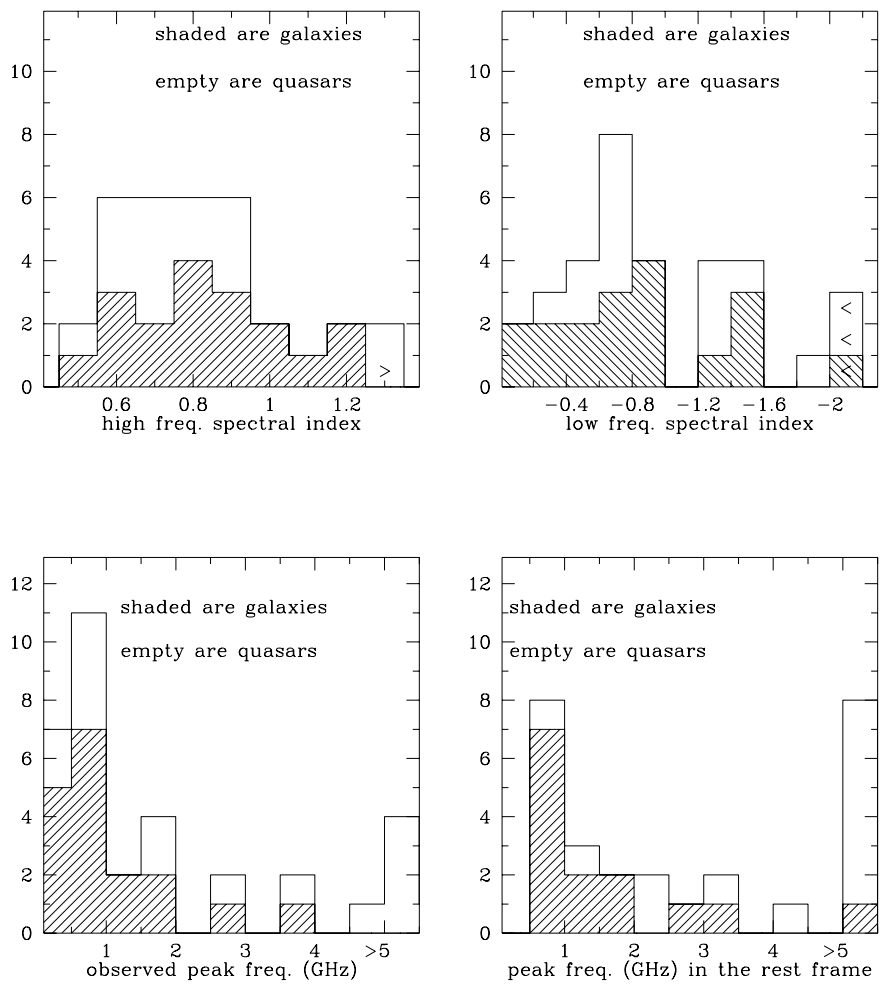

Fig. 6. Histograms for the complete sample: a) high frequency spectral index distribution; b) low frequency spectral index distribution; c) observed turnover frequency distribution; d) rest frame turnover frequency distribution

fainter sample. In addition, VLBI images of several sources belonging to the complete sample show that quasars are more compact than galaxies, and in general exhibit different morphologies (Stanghellini et al. 1997).

These results suggest that either GPS galaxies and GPS quasars are different types of objects, or that beaming of compact components plays a role in the quasars (see also O'Dea 1998).

\subsection{Extended emission}

We have detected extended emission (both diffuse and compact) close to the compact radio source in some cases at $21 \mathrm{~cm}$. In the remaining sources, our upper limits on extended emission is typically $1 \mathrm{mJy} /$ beam at $21 \mathrm{~cm}$.

0248+430 (Fig. 7) has a compact emitting region 15 arcsec east of the main component and a hint of weak emission 5 arcsec to the south. $0528+134$ is resolved, showing an extension in the NW direction (Fig. 8). Murphy et al. (1993) present an image of $0738+313$ at $20 \mathrm{~cm}$ showing 2 emitting regions resembling 2 weak hot-spots and lobes on the opposite sides of the dominant component. In our image (Fig. 9) these 2 weak components are almost completely resolved out and only a hint of emission has been detected 30 arcsec north and south of the compact region. $0941-080$ shows a slightly resolved

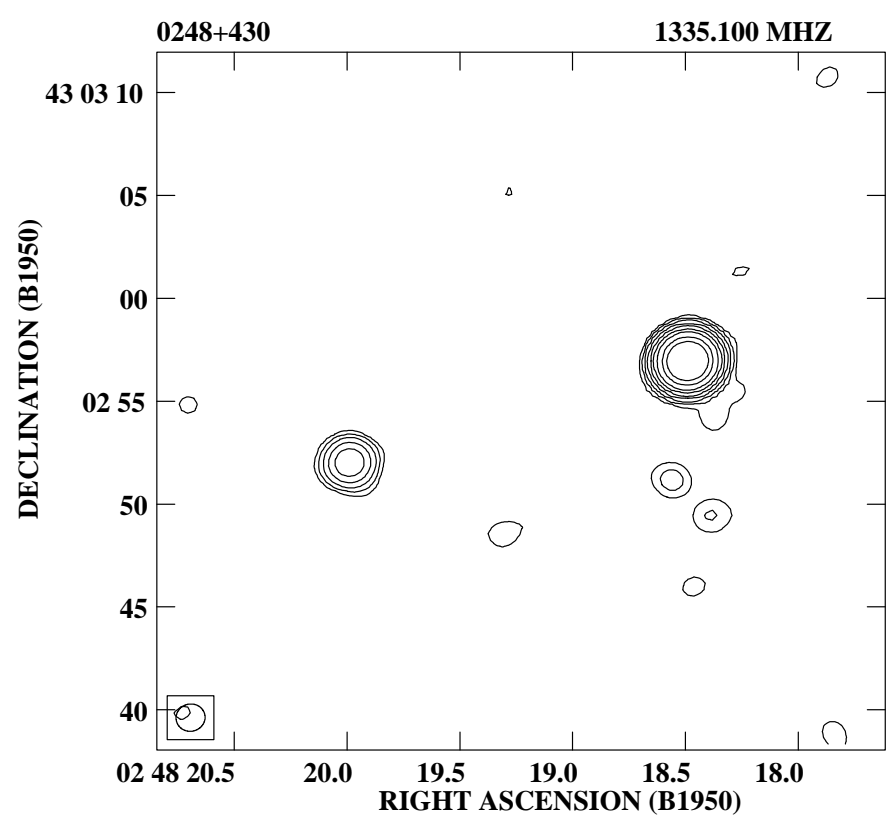

Fig. 7. $0248+430$ at $1.35 \mathrm{GHz}$. The restoring beam is $1.41 \times$ 1.32 arcsec in $\mathrm{PA}-78^{\circ}$. The rms noise on the image is $0.2 \mathrm{mJy}$. The peak flux is $810 \mathrm{mJy} / \mathrm{beam}$. The contour levels for all the images are $-3,3,6,12,25,50,100,200,500,1000 \times$ the $\mathrm{rms}$ noise

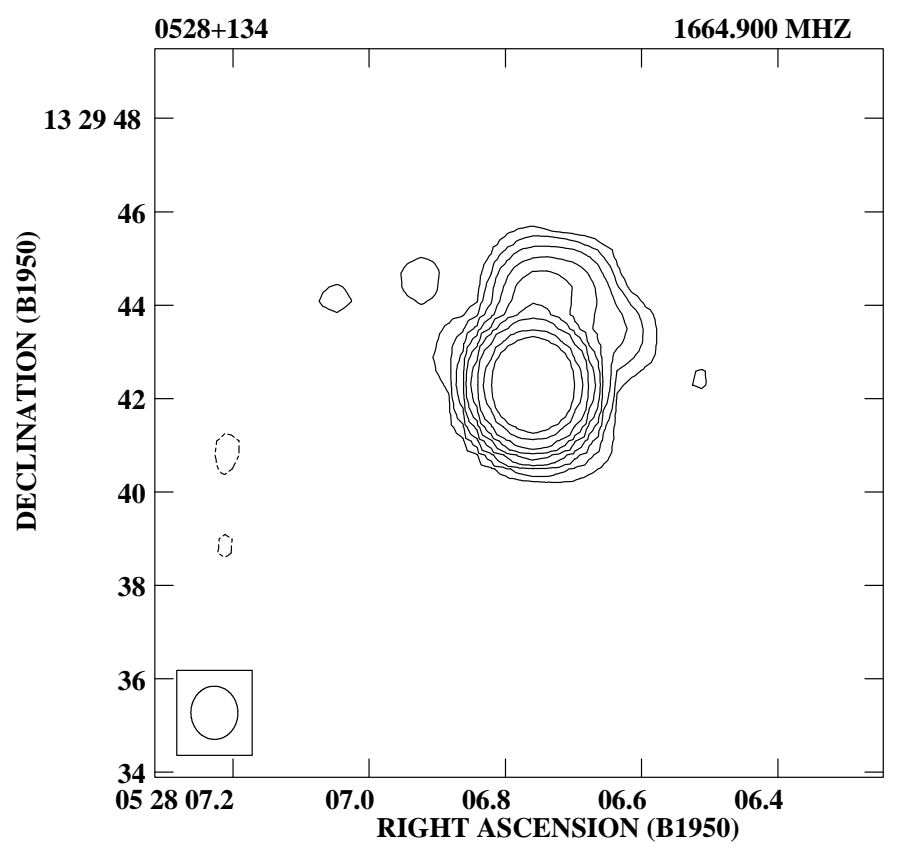

Fig. 8. $0528+134$ at $1.66 \mathrm{GHz}$. The restoring beam is $1.14 \times$ 1.01 arcsec in $\mathrm{PA}+2^{\circ}$. The rms noise on the image is $0.25 \mathrm{mJy}$. The peak flux is $2088 \mathrm{mJy} /$ beam 
Table 4. Polarization for the complete sample

\begin{tabular}{|c|c|c|c|c|c|c|c|c|c|c|c|c|c|c|}
\hline & 1380 & & 1640 & & 4835 & & 4885 & & & & & & & \\
\hline name & $\%$ & $\mathrm{PA}$ & $\%$ & $\mathrm{PA}$ & $\%$ & $\mathrm{PA}$ & $\%$ & $\mathrm{PA}$ & & & & & & \\
\hline 0019-000 & 0.5 & +0 & 0.3 & -2 & $<0.1$ & & $<0.1$ & & & & & & & \\
\hline $0237-233$ & 2.0 & +7 & 1.2 & +4 & 4.0 & -35 & 4.0 & -35 & & & & & & \\
\hline \multirow[t]{2}{*}{$0500+019$} & $<0.2$ & & $<0.2$ & & $<0.1$ & & $<0.1$ & & & & & & & \\
\hline & 1380 & & 1630 & & 4815 & & 4865 & & 8435 & & 8485 & & & \\
\hline name & $\%$ & PA & $\%$ & PA & $\%$ & PA & $\%$ & $\mathrm{PA}$ & $\%$ & $\mathrm{PA}$ & $\%$ & $\mathrm{PA}$ & & \\
\hline $0108+388$ & $<0.2$ & & 0.5 & & $<0.2$ & & $<0.1$ & & $<0.1$ & & $<0.1$ & & & \\
\hline $0316+161$ & 0.5 & -63 & 0.7 & -60 & $<0.1$ & & $<0.1$ & & $<0.1$ & & $<0.1$ & & & \\
\hline $0428+205$ & 0.4 & -59 & 0.6 & -58 & $<0.1$ & & $<0.1$ & & $<0.1$ & & $<0.1$ & & & \\
\hline $0710+439$ & $<0.1$ & & $<0.3$ & & $<0.1$ & & $<0.1$ & & $<0.1$ & & $<0.1$ & & & \\
\hline $1031+567$ & $<0.2$ & & $<0.2$ & & $<0.3$ & & $<0.2$ & & $<0.2$ & & $<0.2$ & & & \\
\hline $1323+321$ & 0.3 & -35 & 0.4 & -01 & $<0.1$ & & $<0.1$ & & 0.9 & +14 & 0.8 & +15 & & \\
\hline $1404+286$ & 0.4 & -50 & $<0.3$ & -23 & $<0.2$ & & $<0.1$ & & $<0.2$ & & $<0.2$ & & & \\
\hline $1518+047$ & $<0.2$ & & $<0.2$ & & $<0.3$ & & $<0.1$ & & $<0.2$ & & $<0.2$ & & & \\
\hline $1607+268$ & $<0.3$ & & $<0.2$ & & $<0.1$ & & $<0.1$ & & $<0.1$ & & $<0.1$ & & & \\
\hline $2008-068$ & 1.7 & -19 & 2.5 & -21 & $<0.3$ & & $<0.3$ & & $<0.3$ & & $<0.3$ & & & \\
\hline \multirow[t]{3}{*}{$2352+495$} & $<0.2$ & & $<0.3$ & -34 & $<0.1$ & & $<0.1$ & & $<0.1$ & & $<0.1$ & & & \\
\hline & 1335 & & 1665 & & 4535 & & 4985 & & 8085 & & 8465 & & $\mathrm{RM}\left(\mathrm{rad} / \mathrm{m}^{2}\right)$ & \\
\hline & $\%$ & $\mathrm{PA}$ & $\%$ & $\mathrm{PA}$ & $\%$ & $\mathrm{PA}$ & $\%$ & $\mathrm{PA}$ & $\%$ & $\mathrm{PA}$ & $\%$ & $\mathrm{PA}$ & obs & rest \\
\hline $0248+430$ & 1.7 & $+52 ?$ & 1.5 & +23 & 2.0 & -18 & 2.0 & -27 & 0.5 & -40 & 0.4 & -44 & 131 & 703 \\
\hline $0457+024$ & $<0.1$ & & $<0.1$ & & $<0.3$ & & 0.3 & +47 & 1.0 & +14 & 1.2 & +12 & 258 & 2954 \\
\hline $0738+313$ & $<0.1$ & & $<0.3$ & & 1.7 & +41 & 1.6 & +67 & 3.0 & -2 & 3.2 & +2 & -813 & -2160 \\
\hline $0742+103$ & $<0.2$ & & $<0.2$ & & $<0.1$ & & $<0.2$ & & $<0.1$ & & $<0.1$ & & & \\
\hline $0743-006$ & 0.4 & -76 & 0.5 & -7 & $<0.2$ & & $<0.2$ & & 0.8 & +1 & 1.0 & -18 & & \\
\hline $0941-080$ & $<0.2$ & & $<0.1$ & & $<0.1$ & & $<0.1$ & & $<0.2$ & & $<0.2$ & & & \\
\hline $1117+146$ & $<0.2$ & & $<0.1$ & & $<0.2$ & & $<0.3$ & & $<0.2$ & & $<0.2$ & & & \\
\hline $1127-145$ & 4.3 & -84 & 3.9 & +61 & 2.6 & -26 & 2.8 & -24 & 3.3 & -17 & 3.3 & -18 & -49 & -234 \\
\hline $1143-245$ & 1.9 & +72 & 2.1 & +80 & 0.7 & -18 & 0.7 & -27 & 1.3 & -43 & 1.4 & -46 & 146 & 1270 \\
\hline $1245-197$ & $<0.2$ & & $<0.1$ & & $<0.1$ & & $<0.1$ & & $<0.1$ & & $<0.1$ & & & \\
\hline $1345+125$ & $<0.2$ & & $<0.1$ & & $<0.1$ & & $<0.2$ & & $<0.1$ & & $<0.1$ & & & \\
\hline $1358+624$ & $<0.2$ & & $<0.1$ & & $<0.1$ & & $<0.3$ & & $<0.2$ & & $<0.2$ & & & \\
\hline $1442+101$ & 0.8 & -74 & 0.8 & -78 & 2.0 & +60 & 1.7 & +64 & 1.4 & +82 & 1.6 & +78 & -116 & -2395 \\
\hline $1600+335$ & $<0.2$ & & $<0.1$ & & $<0.1$ & & $<0.1$ & & $<0.1$ & & $<0.1$ & & & \\
\hline $2126-158$ & $<0.2$ & & $<0.2$ & & $<0.1$ & & $<0.2$ & & $<0.1$ & & $<0.2$ & & & \\
\hline $2128+048$ & $<0.1$ & & $<0.1$ & & $<0.1$ & & $<0.1$ & & $<0.2$ & & $<0.2$ & & & \\
\hline $2134+004$ & $<0.1$ & & $<0.1$ & & 0.9 & +60 & 0.9 & +43 & 0.5 & -4 & 0.5 & +0 & 349 & 3008 \\
\hline $2210+016$ & $<0.1$ & & $<0.1$ & & $<0.1$ & & $<0.1$ & & $<0.2$ & & $<0.2$ & & & \\
\hline $2342+821$ & $<0.2$ & & $<0.1$ & & $<0.1$ & & 0.5 & -63 & & & & & & \\
\hline
\end{tabular}

secondary component 20 arcsec east of the main one (Fig. 10). $2134+004$ has very weak and diffuse emission around the strong compact component (Fig. 11). 2223+210 has a secondary component 4 arcsec away from the main one in the SW direction in our image at $1.35 \mathrm{GHz}$ (Fig. 12); the main component itself is resolved in a core-jet structure oriented NE with a possible counter jet in the image at 5 $\mathrm{GHz}$ (Fig. 13). 2230+114 at $1.35 \mathrm{GHz}$ (Fig. 14) shows an elongated structure in the NW-SE direction with a hint of emission bending to SW, while in the $4.9 \mathrm{GHz}$ image (Fig. 15) the elongated structure turns out to be a core-jet structure with the possible presence of a counter jet.
Stanghellini et al. (1990) report several cases of extended emission around GPS radio sources. Of the objects presented here showing extended emission, 0528+134 and $2223+210$ are not true GPS objects (see also Sect. 4.4 for a discussion of the case of $0528+134)$. In a couple of sources $(0248+430,0941-080$ both belonging to the complete sample) it is difficult to say whether the secondary emission is related to the GPS radio source and further observations are probably needed. The extended emission found around $0738+313,2134+004$, and $2230+114$ (the first 2 objects belong to the complete sample) is likely to be related to the GPS object. In the complete sample, 
Table 5. Polarization of additional objects

\begin{tabular}{|c|c|c|c|c|c|c|c|c|c|c|c|c|c|c|}
\hline & 1335 & & 1665 & & 4535 & & 4985 & & 8085 & & 8465 & & $\mathrm{RM}\left(\mathrm{rad} / \mathrm{m}^{2}\right)$ & \\
\hline name & $\%$ & $\mathrm{PA}$ & $\%$ & $\mathrm{PA}$ & $\%$ & PA & $\%$ & $\mathrm{PA}$ & $\%$ & $\mathrm{PA}$ & $\%$ & $\mathrm{PA}$ & obs & rest \\
\hline $0404+768$ & $<0.2$ & & $<0.1$ & & $<0.1$ & & $<0.3$ & & & & & & & \\
\hline $0440-003$ & 0.9 & +49 & 0.9 & -27 & 2.3 & +61 & 2.1 & +58 & 2.6 & +55 & 2.6 & +56 & 28 & \\
\hline $0528+134$ & 0.6 & -56 & $<0.3$ & & 2.2 & -31 & 2.6 & -33 & 3.9 & -39 & 4.0 & -41 & 52 & \\
\hline $2149+056$ & $<0.2$ & & $<0.2$ & & $<0.1$ & & $<0.1$ & & $<0.2$ & & $<0.2$ & & & \\
\hline $2223+210$ & 13.3 & -28 & 10.5 & -82 & 10.0 & -64 & 9.1 & -58 & & & & -123 & -1077 & \\
\hline \multirow[t]{3}{*}{$2230+114$} & 2.5 & -87 & 2.6 & -33 & 1.2 & +61 & 1.5 & +75 & & & & -59 & -244 & \\
\hline & 1380 & & 1630 & & 4815 & & 4865 & & 8435 & & 8485 & & $\mathrm{RM}\left(\mathrm{rad} / \mathrm{m}^{2}\right)$ & \\
\hline & $\%$ & $\mathrm{PA}$ & $\%$ & $\mathrm{PA}$ & $\%$ & $\mathrm{PA}$ & $\%$ & $\mathrm{PA}$ & $\%$ & $\mathrm{PA}$ & $\%$ & $\mathrm{PA}$ & obs & rest \\
\hline $0026+346$ & 0.4 & -45 & 0.6 & -43 & $<0.2$ & & $<0.2$ & & $<0.1$ & & $<0.1$ & & & \\
\hline $0201+113$ & 0.7 & -48 & 0.9 & -38 & 1.4 & +33 & 1.4 & +33 & 0.5 & +4 & 0.5 & +5 & 131 & 2724 \\
\hline $0552+398$ & 0.4 & -52 & 1.0 & +78 & 0.8 & +43 & 0.6 & +53 & 0.8 & +66 & 0.8 & +66 & -1344 & -15218 \\
\hline $0703+468$ & $<0.1$ & & $<0.2$ & & $<0.2$ & & $<0.2$ & & $<0.3$ & & $<0.3$ & & & \\
\hline $0711+356$ & 1.3 & +5 & 1.0 & -33 & 1.8 & +83 & 1.8 & +80 & 1.9 & +76 & 2.0 & +79 & 40 & 275 \\
\hline $0904+039$ & $<0.2$ & & $<0.2$ & & $<0.5$ & & $<0.5$ & & $<0.9$ & & $<0.9$ & & & \\
\hline $0914+114$ & $<0.2$ & & $<0.2$ & & $<0.8$ & & $<0.8$ & & $<2.0$ & & $<2.0$ & & & \\
\hline $1543+005$ & $<0.1$ & & $<0.2$ & & $<0.2$ & & $<0.2$ & & $<0.2$ & & $<0.1$ & & & \\
\hline $1732+094$ & $<0.2$ & & $<0.3$ & & 1.5 & +16 & 1.4 & -1 & $<0.3$ & & $<0.3$ & & & \\
\hline $2015+657$ & 4.5 & -53 & 3.6 & -71 & 3.2 & +54 & 3.1 & +55 & 4.3 & +51 & 4.3 & +51 & 30 & \\
\hline $2021+614$ & 0.6 & -53 & 1.0 & -51 & $<0.2$ & & $<0.2$ & & $<0.1$ & & $<0.1$ & & & \\
\hline $2050+364$ & $<0.2$ & & 0.4 & -77 & $<0.2$ & & $<0.1$ & & $<0.1$ & & $<0.1$ & & & \\
\hline $2137+209$ & $<0.2$ & & $<0.1$ & & $<0.2$ & & $<0.2$ & & 0.4 & +68 & $<0.3$ & & & \\
\hline $2337+264$ & $<0.2$ & & $<0.3$ & & $<0.3$ & & $<0.2$ & & $<0.2$ & & $<0.2$ & & & \\
\hline
\end{tabular}

$0108+388$ is known to have extended emission, so there are 3 to 5 objects out of 33 with known extended emission so far. This percentage of 9 to $15 \%$ is slightly smaller but consistent with that previously claimed by Stanghellini et al. (1990). It is clear that the vast majority $(\sim 90 \%)$ of the GPS sources appear to be truly isolated and have no emission beyond the kpc scale at the current limits.

\subsection{Variability}

Waltman et al. (1991) presented monitoring observations at 2.7 and $8.1 \mathrm{GHz}$ for several GPS sources covering the time range from 1979 to 1988 . Some sources as $0237-233$, $1245-197,1345+125$ were found to be very stable in flux density. Others were found to be variable: $0552+398$ shows a variation of $\sim 30 \%$ at $8.1 \mathrm{GHz} .2134+004$ has a variability of about $15-20 \%$ at $8.1 \mathrm{GHz} .2352+495$ has a variability below $10 \%$ at $8.1 \mathrm{GHz}$. Also $0742+103$ is slightly variable.

Wehrle et al. (1992) also report variability for some GPS objects in the time range 1985-1991 at 4.8, 8, and $14.5 \mathrm{GHz}$, from the University of Michigan Radio Astronomy Observatory monitoring program for several sources, some of which are GPS objects. $0552+398$ shows an increase in flux density exceeding $50 \%$ at 8.4 and 14.5 $\mathrm{GHz}, 1127-145$ shows a quasi periodical variability of approximately $1 \mathrm{Jy}$ at all the 3 frequencies. The source $2230+114$ also shows a rather remarkable flux density variability at all the 3 frequencies with an amplitude of 0.5 $-1 \mathrm{Jy}$ and is a well known low frequency variable source (Bondi et al. 1996). The variability of $1404+286$ has been discussed by Stanghellini et al. (1996).

In conclusion, we find that some GPS sources (mainly quasars) show mild to high flux density variability at $\mathrm{cm}$ and $\mathrm{mm}$ wavelengths. However, without uniform monitoring of the complete sample it will not be possible to determine how common this variability is. We also note a couple sources where the spectral shape is variable and at some times the spectrum was peaked, and at other times it was not, $0528+134$, the well known gamma-ray source (Mukherjee et al. 1996), and $0201+110$. Both of these sources show a rather flat spectrum in the VLA observations from the second or the third session and they would be very easily discarded as GPS radio sources. But $0528+134$ has been included in the class of GPS radio sources because of its GPS-like spectrum from the literature (O'Dea et al. 1991), and $0201+113$ really shows a convex spectrum in the data in the first VLA session published in O'Dea et al. (1990). This behavior is not surprising in highly variable radio sources as we may well expect that the presence of new radio components will change the spectral shape. Thus, there are sources which show a peaked spectrum only part of the time. This aspect of the 


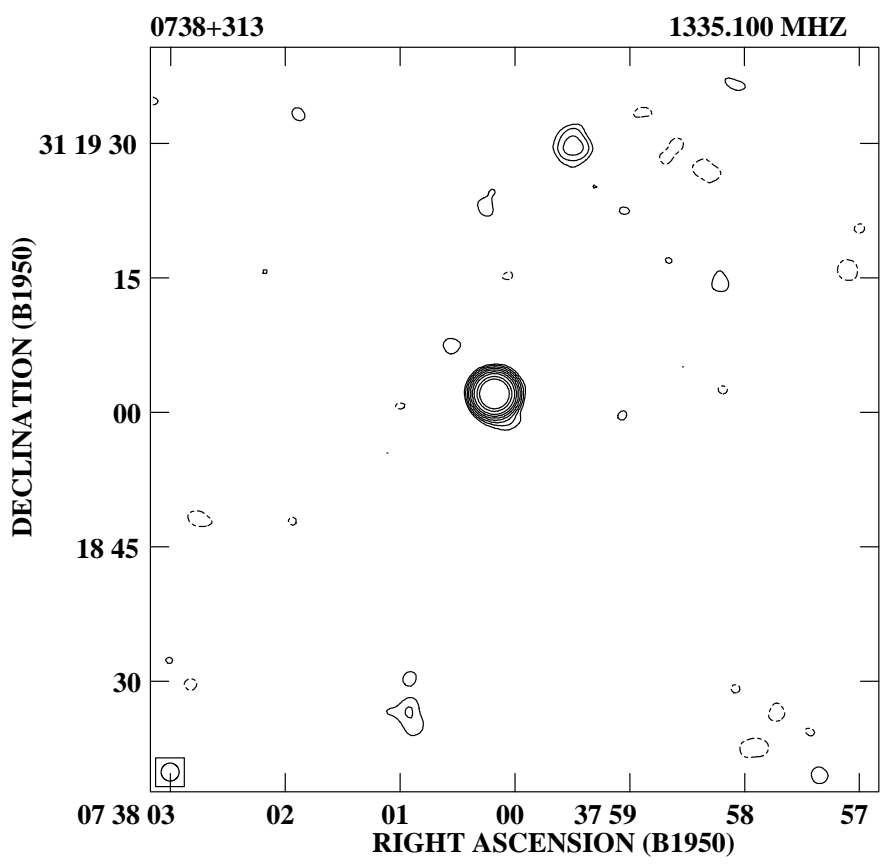

Fig. 9. $0738+313$ at $1.33 \mathrm{GHz}$. The restoring beam is $2 \times$ 2 arcsec. The rms noise on the image is $0.3 \mathrm{mJy}$. The peak flux is $1943 \mathrm{mJy}$

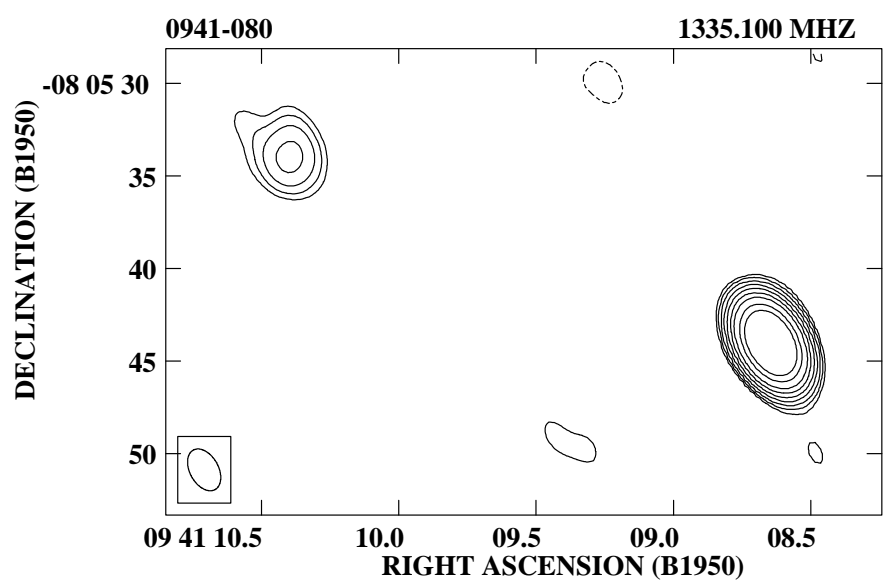

Fig. 10. $0941-080$ at $1.33 \mathrm{GHz}$. The restoring beam is $2.44 \times$ 1.54 arcsec in $\mathrm{PA}+29^{\circ}$. The rms noise on the image is $0.5 \mathrm{mJy}$. The peak flux is $2088 \mathrm{mJy} /$ beam

GPS phenomenon deserves more attention as it could be related to the remarkably different properties found between GPS galaxies and (some?) GPS quasars.

\subsection{Polarization}

Due to the low level of observed polarization, the errors are dominated by the rms noise on the polarization images (typically $0.5 \mathrm{mJy}$ ) and by contamination from residual unpolarized emission (estimated about $0.2-0.3 \%$ of the total flux density). The error in the polarized flux may then be calculated as $\sigma_{\mathrm{P}}=\sqrt{0.5^{2}+\left(0.003 \times S_{\mathrm{mJy}}\right)^{2}} \mathrm{mJy}$.

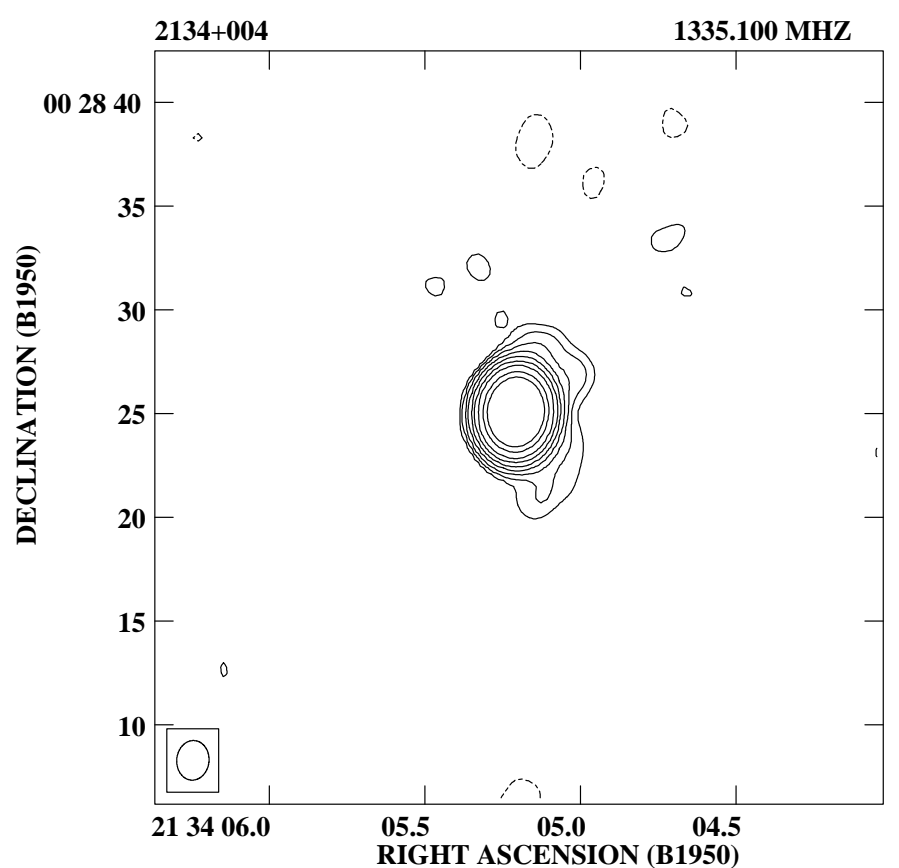

Fig. 11. $2134+004$ at $1.33 \mathrm{GHz}$. The restoring beam is $1.91 \times$ $1.55 \operatorname{arcsec}$ in PA $-5^{\circ}$. The rms noise on the image is $0.4 \mathrm{mJy}$. The peak flux is $3249 \mathrm{mJy} /$ beam

We considered any measurement of the fractional polarization below $0.3 \%$ to be an upper limit (Tables 6 and 7 ). The errors in the position angle have a systematic contribution due to the uncertainty in the determination of the position angle of the calibrator (3C 286), assumed to be around $2-3$ degrees. This is the dominant contribution in the position angle error for most of the objects with detected polarized flux density.

In Fig. 16 we show the histograms of the fractional of polarization (mostly upper limits) for the complete sample at 1.3, 4.9 and $8.5 \mathrm{GHz}$. The fractional polarization is in general low at all the frequencies. Only a few quasars have a fractional polarization above $1 \%$ at 4.9 or $8.5 \mathrm{GHz}$.

When polarized emission has been detected we attempted a linear fit to the polarization angles versus the squared observed wavelength, as is expected from the Faraday effect on polarized radiation propagating through a magnetized and ionized medium.

The low level or even the lack of detection of polarized flux limited us to only a few sources (all quasars). The fits are generally rather good and are given in Tables 6 and 7, and in Figs. 15 and 16. Due to the better frequency coverage in the present observations, our estimated rotation measures supersede those reported by O'Dea et al. (1990), though we cannot rule out that some of the difference is due to variability. We find Faraday rotation measures in the rest frame above $1000 \mathrm{rad} / \mathrm{m}^{2}$ for 5 quasars of the complete sample. We also found a very high value $\left(>10^{4} \mathrm{rad} / \mathrm{m}^{2}\right)$ for $0552+398$ which does not belong to the complete sample but has a GPS shape. 


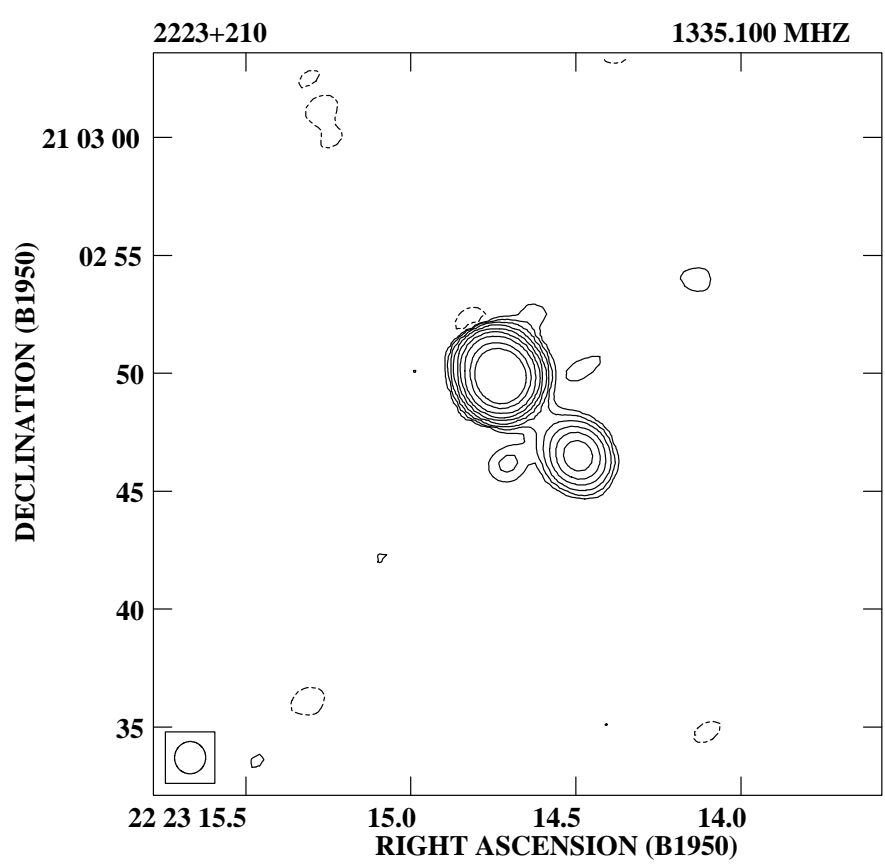

Fig. 12. $2223+210$ at $1.33 \mathrm{GHz}$. The restoring beam is $1.37 \times$ $1.32 \operatorname{arcsec}$ in $\mathrm{PA}-7^{\circ}$. The rms noise on the image is $0.3 \mathrm{mJy}$. The peak flux is $1663 \mathrm{mJy} /$ beam

Sometimes the frequencies which give a good fit include those close to the turnover, and in the case of $0552+398$ are all below the turnover. This implies that the region emitting the polarized emission is different from that responsible for the optically thick emission or that the turnover is not caused by synchrotron self absorption.

\subsection{Summary and conclusions}

We have presented a bright flux-density-limited complete sample of 33 GPS radio sources selected on the basis of their peaked radio spectra. The sample selection was based on observations with the VLA, WSRT, and other instruments. Additional GPS sources not belonging to the complete sample have also been observed. We present our results on the polarization and radio spectrum. We found remarkable differences in the properties of quasars and galaxies, the latter having lower turnover frequencies, mostly undetectable polarization and lower redshifts.

In the few objects where polarization has been detected at many frequencies, the Faraday rotation measure in the rest frame often exceeds $1000 \mathrm{rad} / \mathrm{m}^{2}$.

In about $10 \%$ of the sources we detect weak diffuse extended emission. In the remaining $\sim 90 \%$ any extended emission has a peak surface brightness is less than about $1 \mathrm{mJy} /$ beam at $21 \mathrm{~cm}$.

In a following paper we will discuss the implications of the properties of the complete sample in the framework of the scenarios proposed to explain the existence of the GPS radio sources.

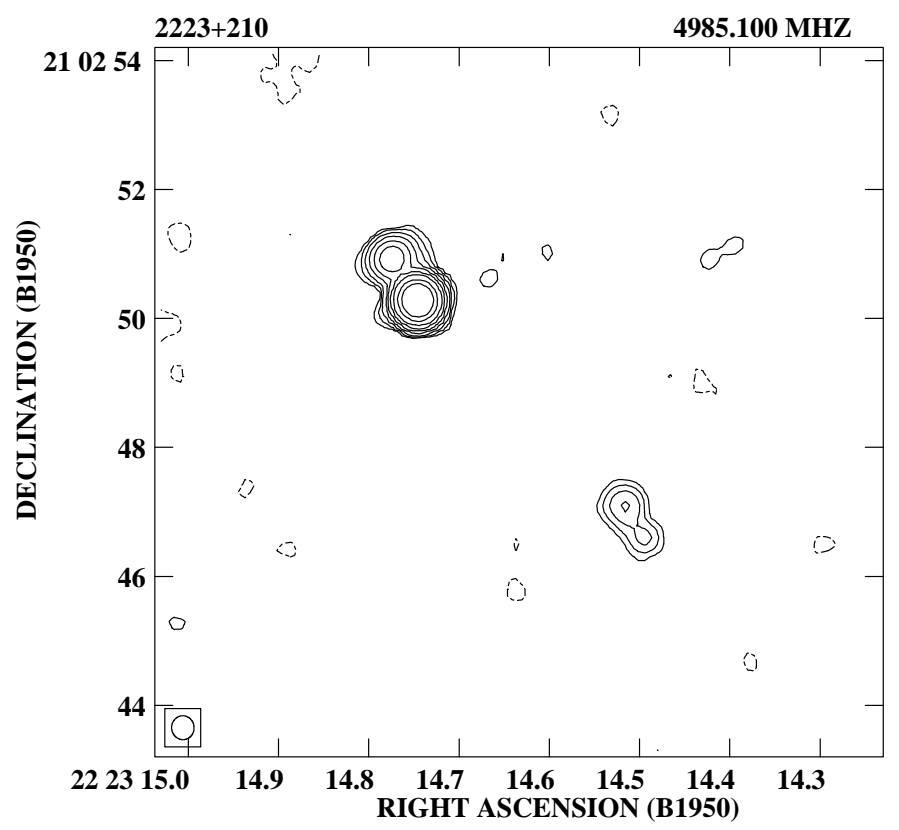

Fig. 13. $2223+210$ at $5 \mathrm{GHz}$. The restoring beam is $0.37 \times 0.35$ arcsec in $\mathrm{PA}+5^{\circ}$. The rms noise on the image is $0.3 \mathrm{mJy}$. The peak flux is $1034 \mathrm{mJy} /$ beam

Acknowledgements. We thank Ger de Bruyn for advice on the reduction of the WSRT observations and Wim De Vries for comments on the manuscript. C.S. wishes to thank the STScI Collaborative Visitor Program for providing support for his visits. The VLA is operated by the U.S. National Radio Astronomy Observatory which is operated by Associated Universities, Inc., under cooperative agreement with the National Science Foundation. The Westerbork Synthesis Radio Telescope is operated by the Netherlands Foundation for Research in Astronomy (NFRA) which is financially supported by the Netherlands organization for scientific research (NWO) in the Hague. We have made use of the NASA/IPAC Extragalactic Database, operated by the Jet Propulsion Laboratory, California Institute of Technology, under contract with NASA.

\section{References}

Baars J.W.M., Genzel R., Pauliny-Toth I.I.K., Witzel A., 1977, A\&A 61, 99

Baldwin J.E., Boysen R.C., Hales S.E.G., Jennings J.E., Waggett P.C., Warner P.J., 1985, MNRAS 217, 717

Baum S.A., O’Dea C.P., Murphy D.W., de Bruyn A.G., 1990, A\&A 23219

Begelman M.C., 1996, in Proceedings of "Cygnus A: Study of a Radio Galaxy," Carilli C. \& Harris D. (eds.). Cambridge, Cambridge University Press, p. 209

Bondi M., Padrielli L., Fanti R., et al., 1996, A\&AS 120, 89

Braccesi A., Ceccarelli M., Colla G., et al., 1969, Nuovo Cimento 62, 13

Carvalho J.C., 1994, A\&A 292, 392

Carvalho J.C., 1998, A\&A 329, 845

Chandler C., 1995, VLA Test Memo 192

Clark B.G., 1980, AA 89, 399 


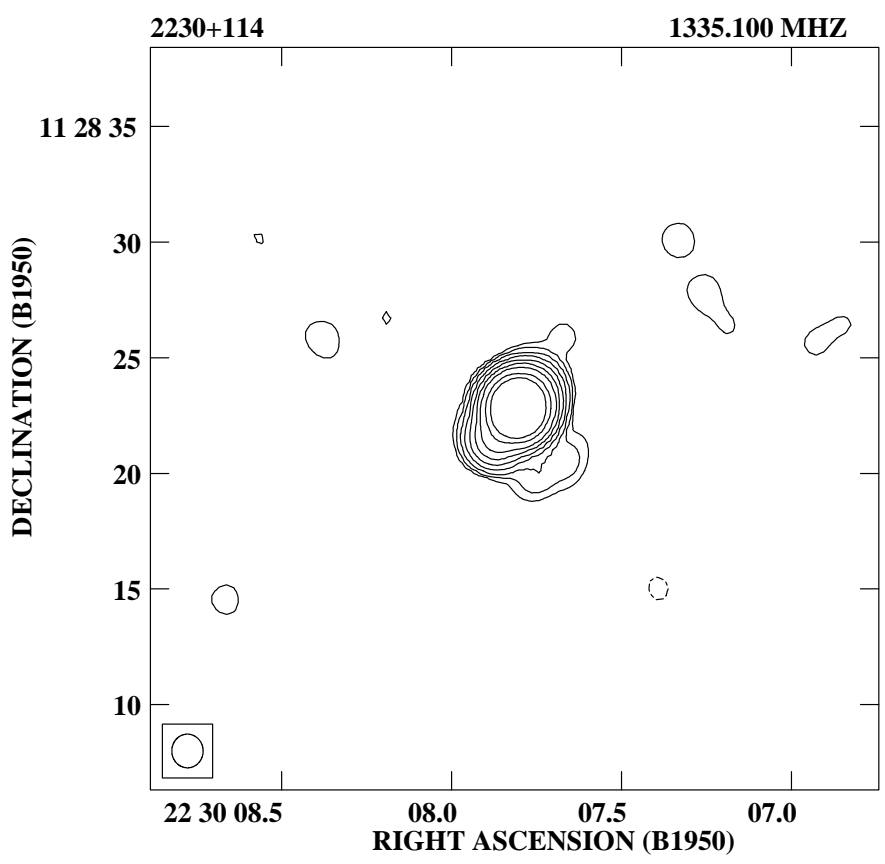

Fig. 14. $2230+114$ at $1.33 \mathrm{GHz}$. The restoring beam is $1.46 \times$ $1.36 \operatorname{arcsec}$ in $\mathrm{PA}+1^{\circ}$. The rms noise on the image is $0.8 \mathrm{mJy}$. The peak flux is $6444 \mathrm{mJy} /$ beam

Cornwell T., Braun R., 1988, in "Synthesis Imaging in Radio Astronomy", Perley R.A., Schwab F.R. and Bridle A.H. (eds.). Astron. Soc. Pac. Conf. Ser. 6, 172

Cornwell T., Fomalont E.B., 1988, in "Synthesis Imaging in Radio Astronomy", Perley R.A., Schwab F.R. and Bridle A.H. (eds.). Astron. Soc. Pac. Conf. Ser. 6, p. 185

Dallacasa D., Stanghellini C., 1990, in Proc. Dwingeloo Workshop, Compact Steep Spectrum and GHz Peaked Spectrum Radio Sources, Fanti C., Fanti R., O’Dea C.P. \& Schilizzi R.T. (eds.). Bologna: Istituto di Radioastronomia, p. 224

de Bruyn A.G., 1990, in Proc. Dwingeloo Workshop, Compact Steep Spectrum and GHz Peaked Spectrum Radio Sources, Fanti C., Fanti R., O'Dea C.P. \& Schilizzi R.T. (eds.). Bologna: Istituto di Radioastronomia, p. 105

Dennison B., Broderick J.J., Ledden J.E., O’Dell, S.L., Condon J.J., 1981, AJ 86, 1604

de Vries W.H., Barthel P.D., Hes R., 1995, AAS 114, 259

de Vries W.H., Barthel P.D., O’Dea C.P., 1997, AA 321, 105

De Young D.S., 1993, ApJ 402, 95

Douglas J.N., Bash F.N., Arakel Bozyan F., Torrence G.W., Wolfe G.W., 1996, AJ 111, 1945

Edelson R.A., 1987, AJ 94, 1150

Esepkina N.A., et al., 1979, Izv. Spets. Astrofiz. Obs. 11, 182

Fanti R., Fanti C., Schilizzi R.T., Spencer R.E., Rendong N., Parma P., van Breugel W.J.M., Venturi T., 1990, AA 231, 333

Fanti C., Fanti R., Dallacasa D., et al., 1995, A\&A 302, 317

Ficarra A., Grueff G., Tomasetti G., 1985, A\&AS 59, 255

Fiedler R.L., Waltman E.B., Spencer J.H., et al., 1987, ApJS 65,319

Gower J.F.R., Scott P.F., Willis D., 1967, Mem. Roy. Astron. Soc. 71,49

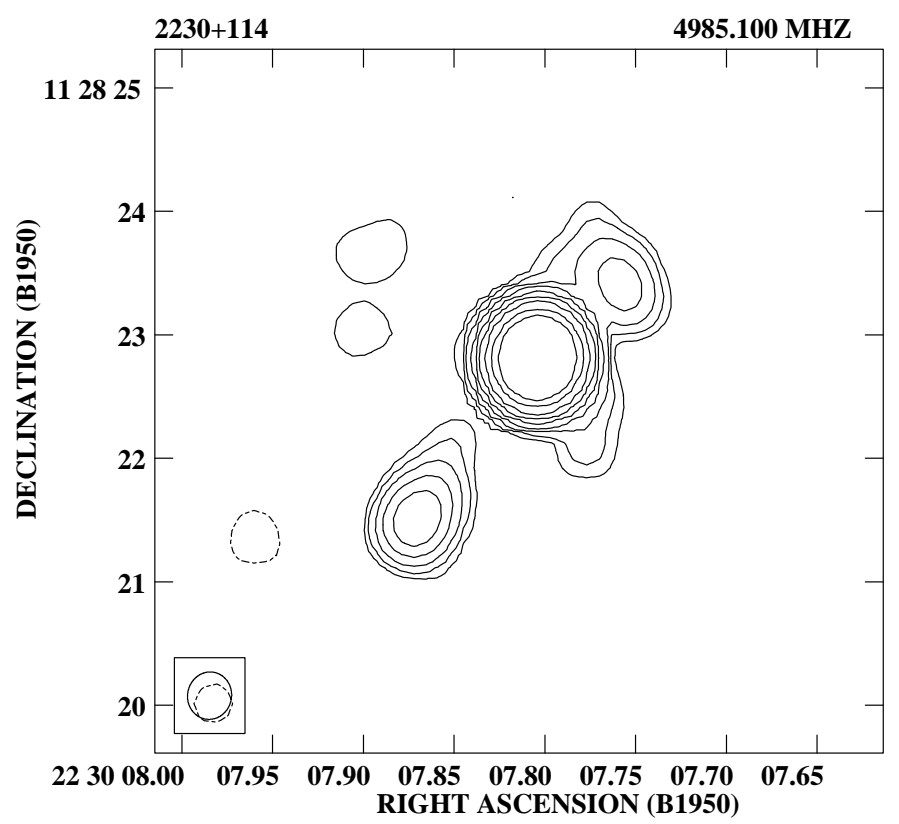

Fig. 15. $2230+114$ at $5 \mathrm{GHz}$. The restoring beam is $0.38 \times 0.36$ $\operatorname{arcsec}$ in $\mathrm{PA}-11^{\circ}$. The rms noise on the image is $0.5 \mathrm{mJy}$. The peak flux is $4137 \mathrm{mJy} /$ beam
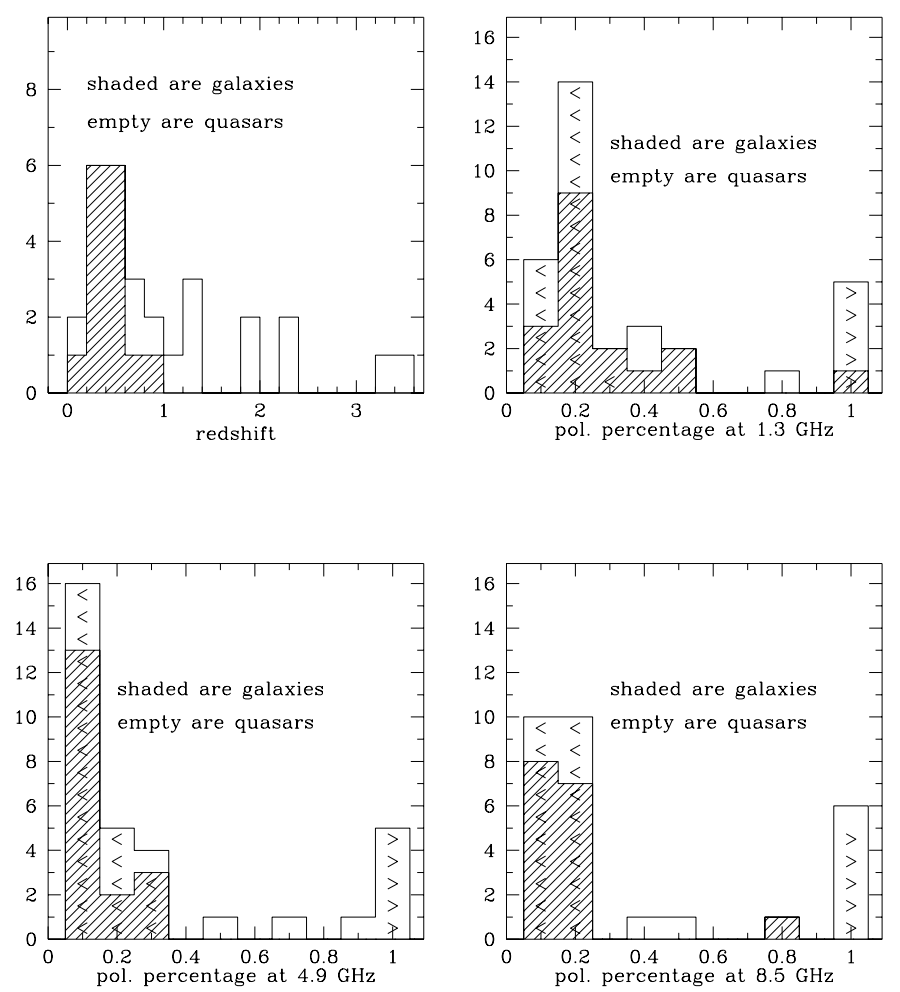

Fig. 16. Histograms for the complete sample: a) redshift distribution; b) fractional polarization at $1.3 \mathrm{GHz}$; c) fractional polarization at $4.9 \mathrm{GHz} ;$ d) fractional polarization at $8.5 \mathrm{GHz}$ 
Hales S.E.G., Baldwin J.E., Warner P.J., 1988, MNRAS 234, 919

Hales S.E.G., Mayer C.J., Warner P.J., Baldwin J.E., 1991, MNRAS 251, 46

Hales S.E.G., Masson C.R., Warner P.J., Baldwin J.E., Green D.A., 1993a, MNRAS 262, 1057

Hales S.E.G., Baldwin J.E., Warner P.J., 1993b, MNRAS 263, 25

Heckman T.M., O’Dea C.P., Baum S.A., Laurikainen E., 1994, ApJ 428, 65

Hewitt A., Burbidge G., 1987, ApJS 63, 1

Hunter S.D., et al., 1993, ApJ 409, 134

Junkkarinen V., Hewitt A., Burbidge G., 1991, ApJS 77, 203

Kühr H., Witzel A., Pauliny-Toth I.I.K., Nauber U., 1981, A\&AS 45, 367

Large M.I., Mills B.Y., Little A.G., Crawford D.F., Sutton J.M., 1981, MNRAS 194, 693

Marscher A.P., 1977, ApJ 216, 244

Mukherjee R., Dingus B.L., Gear W.K., et al., 1996, ApJ 470, 831

Murphy D.W., Browne I.W.A., Perley R.A., 1993, MNRAS 264,298

O’Dea C.P., Baum S.A., Stanghellini C., et al., 1990 A\&AS 84, 549

O'Dea C.P., 1990, MNRAS 245, 20P

O'Dea C.P., Baum S.A., Morris G.B., 1990, A\&AS 82, 261

O'Dea C.P., Baum S.A., Stanghellini C., 1991, ApJ 380, 66

O'Dea C.P., Stanghellini C., Baum S.A., Charlot S., 1996, ApJ 470,806

O'Dea C.P., Baum S.A., 1997, AJ 113, 1480

O'Dea C.P., 1998, PASP (in press)

Phillips R.B., Mutel R.L., 1982, A\&A 106, 21

Pilkington J.D.H., Scott P.F., 1965, Mem. Roy. Astron. Soc. 69, 183

Readhead A.C.S., Taylor G.B., Xu W., Pearson T.J.,
Wilkinson P.N., Polatidis A.G., 1996a, ApJ 460, 612

Readhead A.C.S., Taylor G.B., Pearson T.J., Wilkinson P.N., 1996b, ApJ 460, 634

Riley J.M., 1988, MNRAS 233, 225

Schwab F., 1980, Proc. SPIE 231, 18

Schwab F.R., 1984, AJ 89, 1076

Snellen I.A.G., 1997, PhD thesis

Snellen I.A.G., Bremer M.N., Schilizzi R.T., Miley G.K., van Ojik R., 1996, MNRAS 279, 1302

Stanghellini C., O'Dea C.P., Baum S.A., Fanti R., 1990, in: Proc. Dwingeloo Workshop, Compact Steep Spectrum and GHz Peaked Spectrum Radio Sources, Fanti C., Fanti R., O'Dea C.P. \& Schilizzi R.T. (eds.). Bologna: Istituto di Radioastronomia, p. 17

Stanghellini C., Baum S.A., O'Dea C.P., Morris G.B., 1990, A\&A 233, 379

Stanghellini C., O'Dea C.P., Baum S.A., Laurikainen E., 1993, ApJS 88, 1

Stanghellini C., Bondi M., Dallacasa D., et al., 1996, A\&A 318, 376

Stanghellini C., O’Dea C.P., Baum S.A., et al., 1997, A\&A 325, 943

Steppe H., Salter C.J., Chini R., et al., 1988, A\&AS 75, 317

Steppe H., Liechti S., Mauersberger R., et al., 1992, A\&AS 96, 441

Stickel M., Kühr H., 1993a, AAS 101, 521

Stickel M., Kühr H., 1993b, AAS 100, 395

Teräsranta H., Tornikoski M., Valtaoja E., et al., 1992, A\&AS 94, 121

Waltman E.B., Fiedler R.L., Johnston K.J., et al., 1991, ApJS 77,379

Wehrle A.E., Cohen M.H., Unwin S.C., et al., 1992, ApJ 391, 589

White R.L., Kinney A.L., Becker R.H., 1993, ApJ 407, 456 